\title{
The Motion Path Study of Measuring Robot Based on Variable Universe Fuzzy Control
}

\author{
Ma Guoqing , Yu Zhenglin, Cao Guohua, Zhang Ruoyan and Zheng Yanbin \\ School of Mechatronical Engineering, Changchun University of Science and Technology, Changchun 130022
}

\begin{abstract}
For the problem of measuring robot requires a higher positioning, firstly learning about the error overview of the system, analysised the influence of attitude, speed and other factors on systematic errors. Then collected and analyzed the systematic error curve in the track to complete the planning process. The last adding fuzzy control in both cases, by comparing with the original system, can found that the method based on fuzzy control system can significantly reduce the error during the motion.
\end{abstract}

\section{Introduction}

Robots are complex mechatronic system and easily influenced by external and self structure [1]. Modern control technology for solving robot self adaptation, selflearning ability provides a powerful help. At present, the common robot control technology includes adaptive control, robust control, optimal control, neural network control and sliding mode control.

Chang-Min Ko take a heavy industrial robot as the research object, PI control and fuzzy PD control are studied respectively. The result proves that the fuzzy PD control is more effective [2]. Yu mingliang designed the fuzzy PD control and separate integral control for the mobile robot, simulation results show that the tracking error of the robot becomes very small [3]. The fuzzy control was applied to a three freedom parallel robot by Jingjun Zhang [4]. To prove the control precision of the robot is improved by using fuzzy control, ADAMS and MATLAB joint simulation were used; Gao yanfeng proved that the predictive fuzzy control is more advantage than traditional fuzzy controller by arc seam tracking experiments [5].

\section{Establish a model of measuring robot}

Robot kinematics study the relationship between the the each joint variable and the end position, in order to describe movement or rotation relationship between the joint and the end. Denavit and Hartenberg put forward DH parameter method. This section establishing the robot joint coordinates (as shown in Fig. 1) based on DH parameter method. Its corresponding $\mathrm{DH}$ parameters as shown in Table 1.

$\theta$ is Joint angle, which represents angle between the the $x_{i-1}$ and $x_{i}$ axis about the $z_{i-1}$ axis; $d$ is link offset, which represents the distance from the origin of frame $i-1$ to the $x_{i}$ axis along the $z_{i-1}$ axis;

$\alpha$ is link twist, which represents the angle from $z_{i-1}$ axis to the $z_{i}$ axis about the $x_{i}$ axis;

$a$ is link length, which represents the distance from the the $z_{i-1}$ axis and the $z_{i-1}$ axis along the $x_{i}$ axis, for intersecting axis is parallel to $z_{i-1} \times z_{i}$;

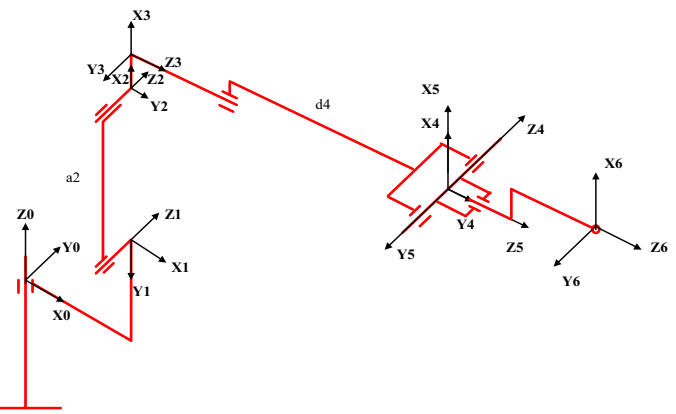

Figure. 1. Measuring robot $\mathrm{DH}$ model.

Table 1. DH parameters.

\begin{tabular}{rcccr}
\hline Axis & $\alpha\left(^{\circ}\right)$ & $a(\mathrm{~m})$ & $\theta\left(^{\circ}\right)$ & $d(\mathrm{~mm})$ \\
\hline 1 & -90 & 0.150 & 0 & 0.173 \\
2 & 0 & 0.600 & -90 & 0.001 \\
3 & -90 & 0.125 & 0 & 0.000 \\
4 & 90 & 0.000 & 0 & 0.640 \\
5 & -90 & 0.000 & 0 & 0.000 \\
6 & 0 & 0.090 & 0 & 0.266 \\
\hline
\end{tabular}

\section{PID parameters optimization based on variable universe fuzzy theory}

For a low stiffness series system such as a measurement robot, with a PID parameters cannot meet the control precision of the system obviously. Controller can adjust PID parameters according to the system overshoot and 
time, steady-state error, and so on, in order to achieve the purpose of optimal control. The detailed process is as follows: the object of fuzzy control is proportional gain $K_{p}$, integral gain $K_{i}$ and differential gain $K_{d}$. For the velocity $K_{v f f}$ and acceleration feed-forward coefficient $K_{\text {aff }}$.After initial position on the robot manually setting will no longer changed.

\subsection{The design of fuzzy controller}

\subsubsection{Language and language variable value}

Fuzzy language is used to describe an object by fuzzy control, fuzzy control objects has 5 . Set the language of error variable is $E$, linguistic variable error rate of change of $E_{c}$, the proportional gain correction amount of linguistic variables is $\Delta K_{p}$, integral gain correction amount of linguistic variables is $\Delta K_{i}$, differential gain correction amount of linguistic variables is $\Delta K_{d}$. They are the domain $\{-3,-2,-1,0,1,2,3\}$ the corresponding language is $\{$ NB NM NS ZO PS PM PB\}, expressed great negative, negative, small medium large zero, forward small medium, large forward great.

\subsubsection{Language membership function values}

Membership function of fuzzy control is mainly used for object describe the extent of the value, the language is related to the actual situation of this system and the objects understanding and judgment of operator. Membership function in compliance with objective reality as the standard,the membership function have many form, commonly used trigonometric functions, Gaussian function and so on, using the trigonometric functions in this project as shown in Fig. 2, the membership function of the specific value show in Table 2:

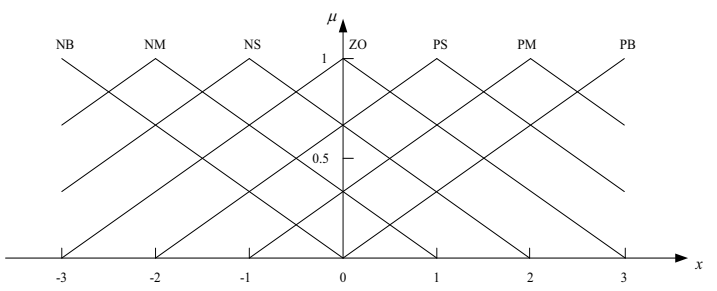

Figure. 2. Triangular membership function

Table 2. Membership function value

\begin{tabular}{|c|c|c|c|c|c|c|c|}
\hline input & -3 & -2 & -1 & 0 & 1 & 2 & 3 \\
\hline $\mathrm{NB}$ & 1 & 0.65 & 0.3 & 0 & 0 & 0 & 0 \\
\hline NM & 0.7 & 1 & 0.65 & 0.35 & 0 & 0 & 0 \\
\hline NS & 0.4 & 0.7 & 1 & 0.65 & 0.35 & 0 & 0 \\
\hline $\mathrm{ZO}$ & 0 & 0.3 & 0.65 & 1 & 0.65 & 0.3 & 0 \\
\hline PS & 0 & 0 & 0.35 & 0.65 & 1 & 0.7 & 0.4 \\
\hline PM & 0 & 0 & 0 & 0.35 & 0.65 & 1 & 0.7 \\
\hline PB & 0 & 0 & 0 & 0 & 0.3 & 0.65 & 1 \\
\hline
\end{tabular}

\subsubsection{Language and language variable value}

Fuzzy rules is composed of a series of fuzzy conditional statement, each statement on behalf of a relationship, such as when the error and error change rate is great, The output of the $\Delta K_{p}$ negative is great, This statement can be represented as IF $\mathrm{E}=\mathrm{PB}$ and $E_{c}=\mathrm{PB}$ THEN=NB . Fuzzy rules to a large extent affected the effect of fuzzy control. Here according to the experience of the engineering practice, set up $\Delta K_{p} \Delta K_{i}$ and $\Delta K_{d}$, fuzzy rule shown in Table 3.

Table 3. Fuzzy rule table of $\Delta K_{p}$

\begin{tabular}{llllllll}
\hline Ec & NB & NM & NS & ZO & PS & PM & PB \\
\hline NB & PB & PB & PM & PM & PS & ZO & ZO \\
NM & PB & PB & PM & PS & PS & ZO & NS \\
NS & PM & PM & PM & PS & ZO & NS & NS \\
ZO & PM & PM & PS & ZO & NS & NM & NM \\
PS & PS & PS & ZO & NS & NS & NM & NM \\
PM & PS & ZO & NS & NM & NM & NM & NB \\
PB & ZO & ZO & NM & NM & NM & NB & NB \\
\hline
\end{tabular}

\subsubsection{Defuzzification}

The result is a collection of fuzzy reasoning, the process of transforming the fuzzy set to the output is called fuzzy solution, fuzzy methods include weighted average method and the maximum membership degree method.Maximum membership degree method, just as its name implies is to maximum membership degree in fuzzy set value corresponding linguistic variables as output.Set the output fields to $A$, then the output value of the maximum membership degree method is :

$$
U=\left\{e^{*} \mid \mu\left(e^{*}\right)>\mu(e) \quad e, e^{*} \in A\right\}
$$

In the actual situation of Chinese style $e^{*}$ may have more than one, then you can take the average or maximum minimum value, this article USES the method of minimum maximum, the description is as follows:

$$
U=\operatorname{Min}\left\{e^{*} \mid \mu\left(e^{*}\right)>\mu(e) e, e^{*} \in A\right\}
$$

\subsubsection{Variable universe factor}

In fuzzy control, as the error decreases, error corresponding language variable values can also reduce accordingly. This will affect the precision of the fuzzy control, but the introduction of the theory of domain factor can contribute to real-time expansion theory domain.Improve the fine degree of control. In the literature [6] puts forward a new theory of variable domain factor,not only few parameters and is also small amount of calculation, front design, with reference to the literatur [7] to be considered at the same time , designed variable domain factors as follows:

$$
\tau(x)=3^{-\lambda}|x|^{\lambda}+\varepsilon \quad(0<\lambda<1)
$$


in the type: $\lambda$-scaling factor parameters, this article take $0.5 \varepsilon$ - the smallest positive number, this article take 0.001 ; Literature pointed out that the theory of variable domain factor in theory domain $U=\left[\begin{array}{ll}3 & 3\end{array}\right]$ need to meet the dual character, avoid zero, monotonicity, coordination and normality. For type (3) the variable domain factor to fully meet the above requirements.

\section{The fuzzy optimization experiments of measuring robot path}

\subsection{The design of fuzzy controller}

In Table 4 for the experiment of four posture of the robot, under the initial posture has completed the system manual adjustment of PID parameters, take 1 shaft, the step response as shown in Fig. 3 (a). Now by robot step response under different attitude to observe attitude of the influence of system error,this article selects three kinds of typical gesture,concrete numerical value shown in table 6.Tests found only 1 shaft has obvious change under the three attitude dynamic performance.

Fig. $3(b) / 3(c) / 3(d)$ in addition to position 3 , the remaining 1 shaft dynamic performance under two kinds of gestures are getting worse, step response show obvious fluctuation.Contrast three kinds of attitude discovery, all dynamic performance with poor posture.

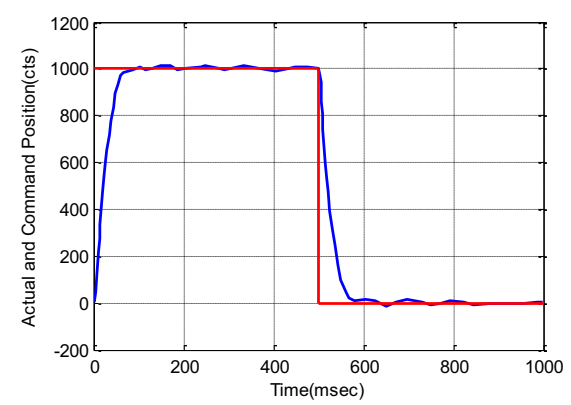

(a) step response of initial posture

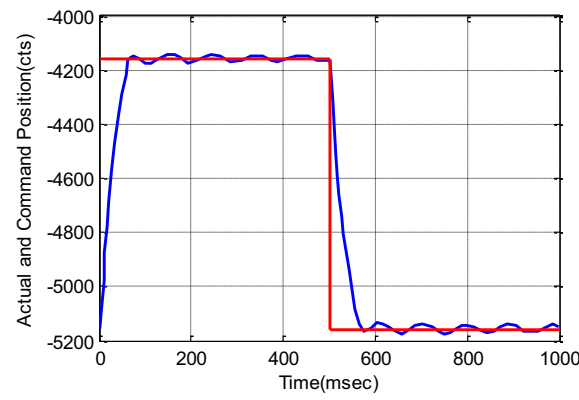

(b) step response of pose 1

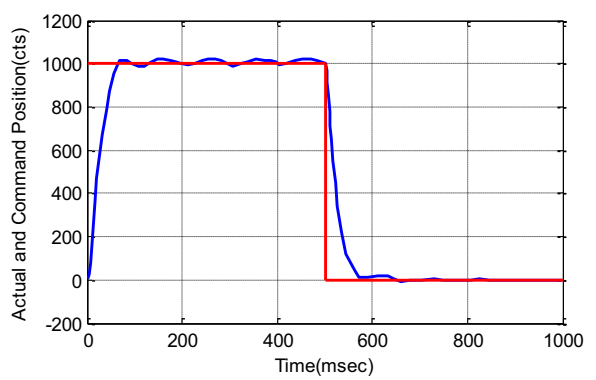

(c) step response of pose 2

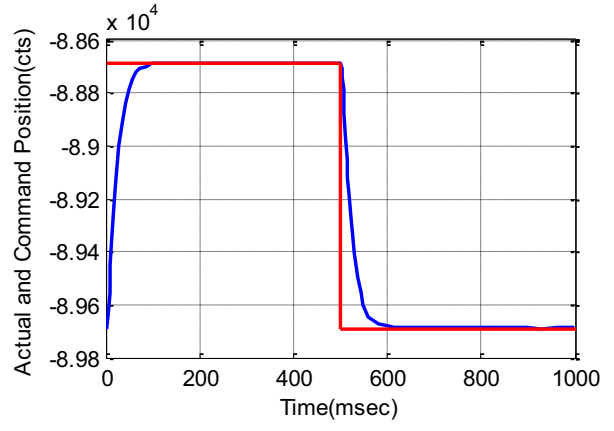

(d) step response of pose 3

Figure. 3. The step response of 1 axis in different posture

Table 4. Pose data of robot(uint $\left(^{\circ}\right)$ ).

\begin{tabular}{lcrrrrr}
\hline pose & A1 & A2 & A3 & A4 & A5 & A6 \\
\hline pose1 & -1.83 & 18.95 & -12.86 & -18.95 & -14.40 & -15.32 \\
pose 2 & 0 & 26.68 & 20.57 & 18.95 & 24.00 & 38.30 \\
pose 3 & -31.81 & -18.95 & 2.57 & -12.63 & 9.60 & 7.66 \\
\hline
\end{tabular}

\subsection{The change of system error in the motion}

From the previous section, the big arm forward 1 shaft dynamic performance is poorer, so when planning robot trajectory. Ensure the protraction of the big arm as far as possible. This paper shows a way to teach the planning of a straight line (two points determine a straight line) and a circular arc (three points to determine a circular arc). Concrete numerical value shown in table 5 and table 6.The speed of the robot is $90 \mathrm{~mm} / \mathrm{s}$ and $70 \mathrm{~mm} / \mathrm{s}$ in the finish line and arc trajectory.

Table 5. Trajectory data of line(uint $\left(^{\circ}\right)$ )

\begin{tabular}{lllrrrrr}
\hline $\begin{array}{lllrrrr}\text { Axis } \\
\text { Point }\end{array}$ & A1 & & A2 & A3 & A4 & A5 & A6 \\
\cline { 1 - 5 } origin & 0 & 0 & 0 & 0 & 0 & 0 \\
Start & -43.24 & 26.22 & 15.21 & -31.01 & -23.79 & 42.27 \\
end & -57.73 & 55.91 & 33.20 & 19.48 & -39.36 & -42.28 \\
\hline
\end{tabular}

Table 6. Trajectory data of circle(uint $\left.\left(^{\circ}\right)\right)$

\begin{tabular}{llrrrrl}
\hline Axis & \multirow{2}{*}{ A1 } & A2 & \multicolumn{1}{l}{ A3 } & A4 & A5 & A6 \\
\hline Point & & 0 & 0 & 0 & 0 & 0 \\
origin & 0 & 0 & 27.83 & 0.01 & -48.67 & 0.02 \\
Apoint & 0.05 & 34.12 & 27.86 & -26.15 & -46.63 & -28.32 \\
Bpoint & -20.50 & 25.56 & 14.09 & 16.152 & -38.89 & -17.66 \\
Cpoint & -11.46 & -3.81 & -20.97 & 10.52 & \\
\hline
\end{tabular}

Fig. 4 and Fig. 5 is spindle motor error variation of 1 shaft in the motion of robot, can see from the diagram, whether a straight line trajectory or circular trajectory, and can match with velocity changes.In the process of robot movement, the following error is the dominance in error sources, and the error is relatively large. Linear and circular arc in the process of movement error range respectively is $[285.83,2443.26]$ and $[226.42,361.73]$, unit is pulse.

Now as an example, analysis the change of the error in a straight line motion, for the convenience of description. Set 1 shaft motor corresponding to the origin, the position of the starting point and end point of A, B, C respectively. As shown in Fig. 5, when the motor 1 received instructions from $\mathrm{A}$ to $\mathrm{B}$, motor acceleration 
instantaneous added to a larger values, making speed up in a short time. The resulting is follow error also rising sharply, when the speed reaches a certain value when the basic remain unchanged.At this point, the following error is similar to the horizontal line, will soon arrived at point B ,speed quickly dropped to zero speed error caused by suddenly disappeared. 1 is the above process in Fig. 7, 2 is 1 shaft spindle motor curves in the process of the robot in a straight line (from B to C), the motor is reverse. The speed is negative. There for when the error is negative, due to the robot slower $(90 \mathrm{~mm} / \mathrm{s})$. At this time of the error value relative to the previous inhibits PTP (A to B) are smaller. The formation of 3 and 1 are similar, just the distance of the robot walk further, speed is relatively large, so the error value also rose slightly.

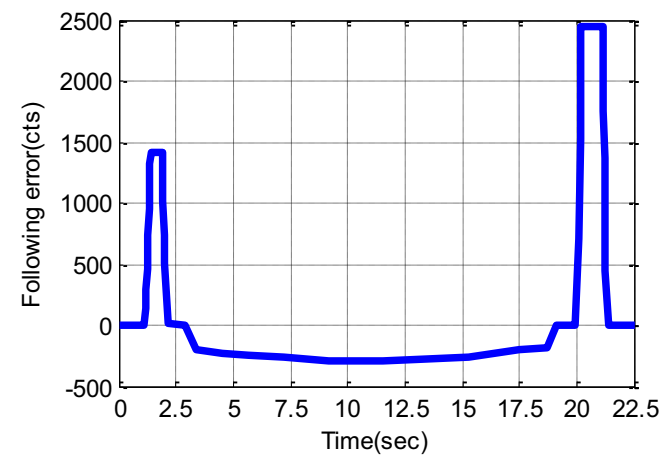

Figure 4. Error variation of 1 axis during linear motion

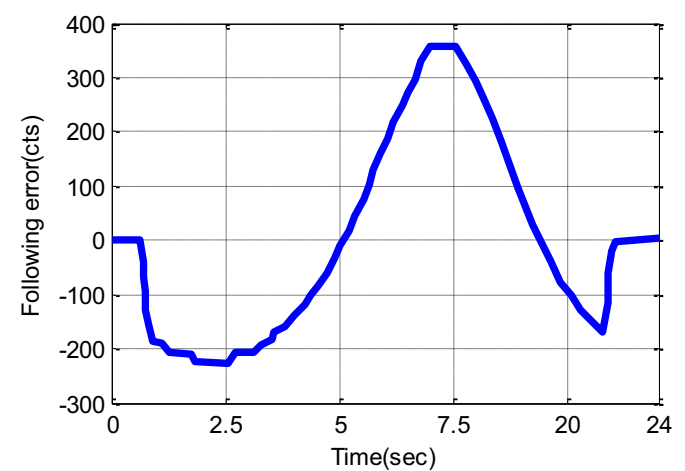

Figure 5. Error variation of 1 axis during arc motion

Through the above analysis that can draw the following conclusion, the following error in the process of movement is the main error; he greater the velocity and acceleration, the greater the error also; the plus or minus of error depend on the direction of speed.

\subsection{Experiment and analysis}

To make up for the defects, this article into the fuzzy adaptive control. Through the optimization of PID parameters to further reduce the error.

Consider safety factors, in practical applications, the PID parameters, set the upper and lower limits, effect of the fuzzy control is shown in Fig. 6 and Fig. 7, you can see that the feedforward, fuzzy control effect is more obvious.

In linear motion, for example, when the error suddenly rises, fuzzy control quickly down the overshoot
(1) in Fig. 6, Once to set the upper and lower limit of PID parameters, PID parameters will not change, the error of line approximation ( 2 in Fig. 6). After movement has stopped, the speed of error caused by rapidly disappearing. At this time of the error is mainly composed of noise, friction factors, such as its value in the range of 3 cts change. Due to the change range is relatively small.Approximation error line is a horizontal line (3) in Fig. 7.

Here it should be pointed out that, the robot in the movement process and motion stops, the main factors causing error are not the same. So use movement at the end of the optimization of the PID parameters to adjust the system error of resting, the effect is not very ideal.This topic research the repositioning precision of the robot in between $0.06 \mathrm{~mm}$, the PID value of manual setting in the system when the speed suddenly 0 means that the error of the effect is satisfying, so when the velocity is zero, PID value is given the system initialization.

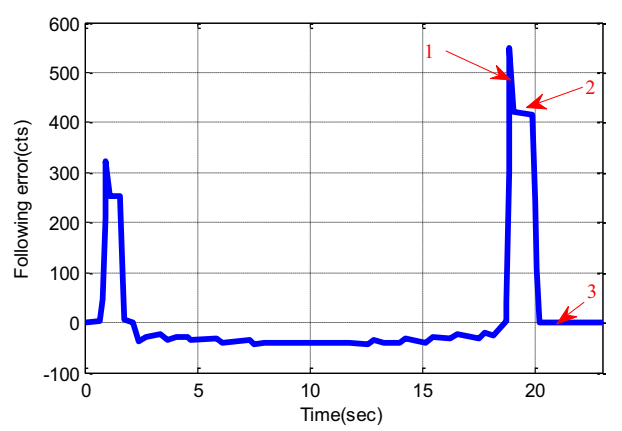

Figure 6. Linear motion error

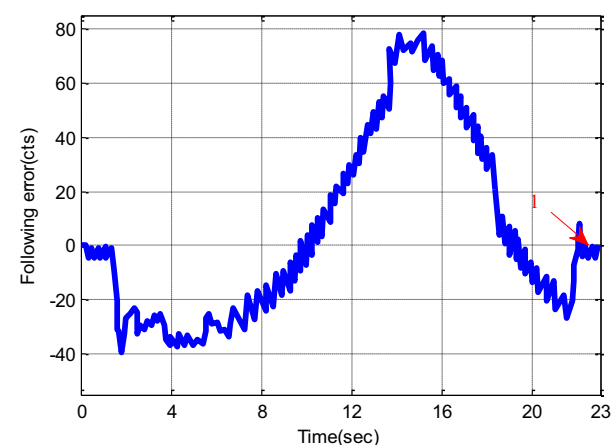

Figure 7. Circular motion error

Mentioned earlier, the PID value is set the upper and lower, many times experiments show that the PID adjustable range is wide, error drop, the more obvious. But if the PID adjustment range is too wide, PID values has far deviated from initial value when speed is zero, if once the speed is zero at this time, PID suddenly give initial value, when system occur large overshoot and oscillation, it will be stabilized at about 20cts even after the shock elimination of error value. This is deadly, because it will greatly influence the positioning precision of the system repeated, so the PID upper and lower's setting needs to be appropriate.After many experiments, can found, when the quantitative factor was set to 0.1 , the scaling factor is set to ,change PID parameters in between, and the tracking accuracy and repeat positioning accuracy 
are in good level (see Table 9 and Table 10).As shown in figure1,because suddenly initialise cause slight overshoot, the super tone value for $9 \mathrm{cts}$, but it can be seen that overshoot is almost just a moment, then the system back to the ideal level (3 cts).Visible is reasonable setting in front of the upper and lower limits. Table 9 and Table 10 is the optimization effect compared with the before.

Table 7. Set of quantization and scaling factor

\begin{tabular}{lccccc}
\hline factor & $\mathrm{E}$ & $\mathrm{E}_{\mathrm{c}}$ & $\Delta K_{p}$ & $\Delta K_{i}$ & $\Delta K_{d}$ \\
\hline vale & 10 & 10 & 1 & 1 & 1 \\
\hline
\end{tabular}

Table 8. PID parameter range of motor 1

\begin{tabular}{rccc}
\hline parameters & $K_{p}$ & $K_{d}$ & $K_{i}$ \\
\hline initial & 290 & 380 & 410 \\
range & {$[220,360]$} & {$[310,450]$} & {$[340,480]$} \\
\hline
\end{tabular}

Table 9. Effect comparison before and after optimization (Line)

\begin{tabular}{cll}
\hline \hline scheme & primeval & fuzzy control \\
$\begin{array}{cll}\text { max error(cts) } \\
\text { process }\end{array}$ & & \\
\hline origin- $>$ Start & 1426.22 & 254.19 \\
Start- $>$ end & -286.18 & -32.22 \\
end- $>$ Start & 2443.26 & 418.27 \\
Speed of 0 & 2.99 & 2.96 \\
\hline
\end{tabular}

Table 10. Effect comparison before and after optimization (circle)

\begin{tabular}{|c|c|c|}
\hline$\frac{\text { scheme }}{\frac{\max \text { error(cts) }}{\text { process }}}$ & primeval & fuzzy control \\
\hline forward direction & 362.33 & 77.62 \\
\hline negative direction & -226.64 & -37.36 \\
\hline Speed of 0 & 2.96 & 2.96 \\
\hline
\end{tabular}

\section{Summary}

Analysised the influence of attitude, speed and other factors on systematic errors, planed the two trajectories (lines and circles), collected and analyzed the systematic error curve in the track to complete the planning process. added fuzzy control in both cases, by comparing with the original system, can found that the method based on fuzzy control system can significantly reduce the error during the motion path. This provide a basis for conduct robot motion path planning and optimization based on fuzzy adaptive control in the future

\section{References}

1. Matveev A S, Hoy M, Katupitiya J, et al. Nonlinear sliding mode control of an unmanned agricultural tractor in the presence of sliding and control saturation. Robotics and Autonomous Systems, 61(9): 973-987, (2013).

2. Chang-Min Ko, Seung-Kyu Park, Tae-SungYoon, et al. Comparative Study of Fuzzy PD Control and PI Control for Heavy Duty Robot. International Conference on Industrial Technology, (2009).

3. Yuming Liang, Lihong $\mathrm{Xu}$, Ruihua Wei, et al. Adaptive Fuzzy Control for Trajectory Tracking of Mobile Robot. International Conference onIntelligent Robots and Systems, Taipei, Taiwan: 4755-4760, (2010).

4. Jingjun Zhang, Chaoyang Lian, Ruizhen Gao, et al. 3-Degree-of-freedom Parallel Robot Control Based Fuzzy Theory. International Conference on Intelligent Human-Machine Systems and Cybernetics: 221-224, (2010).

5. Gao yanfeng, Zhang hua, Mao zhiwei, et al.Predictive Fuzzy Control for a Mobile Welding RobotSeam Tracking. World Congress on Intelligent Control and Automation: 2271-2276, (2008).

6. Cheng Shao, Dong Xiwen, Wang Xiaofang, etc. Variable Universe Fuzzy Controller Selection Factor telescopic. Information and Control, 39(5): 536-541, (2010).

7. Li Hongxing variable universe adaptive fuzzy controller. Science in China: Series E., 29(1): 32-42, (1999). 